\title{
Complete characterization of double photoionization processes
}

\author{
I. A. Ivanov* and A. S. Kheifets \\ Research School of Physical Sciences, The Australian National University, Canberra ACT 0200, Australia
}

(Received 21 March 2011; published 16 June 2011)

\begin{abstract}
We analyze correlated photoelectron spectra of single-photon two-electron ionization [double photoionization (DPI)] of helium to reconstruct the phase of the spectral amplitude of this process. The phase can be reconstructed reliably in a wide range of photoelectron momenta, thus allowing one to retrieve information about the wave function of the DPI process and its temporal evolution. Our simulation indicates that the proposed phase reconstruction technique can be applied in experiment to trace dynamics of the DPI process with attosecond precision.
\end{abstract}

\section{INTRODUCTION}

The method of attosecond pump-probe spectroscopy allows one to get an insight into temporal evolution of atomic and molecular processes on the attosecond time scale [1-3]. By using this technique, it has become possible to observe transient processes [4], electron tunneling [5] in atoms, and probe ultrafast electron motion in low-excited atomic states [6]. The pump-probe approach can also be used to reveal timevarying correlated motion of electrons in the process of double atomic ionization. It was shown [7] that, for the suitably prepared initial wave packets, electron spectra in this process show distinct traces of rotational and vibrational modes in agreement with the well-known view of doubly excited atomic states as states of a floppy triatomic molecule [8,9].

Excellent temporal resolution, which is achieved by using attosecond pump pulses, results in poor energy resolution. Wave packets created by attosecond pulses have a broad spectrum and may include a number of bound states, making detailed analysis difficult [10]. The same problem of broad wave packets and comparatively low spectral resolution hampers the study of temporal evolution of photoionization phenomena based on the time-delay theory $[11,12]$. Techniques such as continuum reference wave-packet [10] or electron wave-packet interference [13] allow one to overcome this problem and to achieve high temporal and spectral resolution simultaneously.

As an alternative to traditional attosecond pump-probe spectroscopy for two-electron ionization processes, which may suffer from poor spectral resolution and low count rate, a measurement scheme was proposed based on correlation between the two emitted photoelectrons [14]. The utility of this scheme was demonstrated in the case of Auger decay triggered by absorption of an extreme ultraviolet (XUV) photon and modulated by a weak infrared (IR) field. By varying the delay between the XUV and IR fields, one can create different patterns of modulation, which can be analyzed to retrieve the phase of the spectral amplitude of the Auger process. This, in turn, can be converted into the timing information to trace the dynamics of the ionization process with attosecond precision.

In this paper, we apply this scheme to the process of single-photon two-electron ionization [double photoionization

*Corresponding author: igor.ivanov@anu.edu.au
(DPI)] of helium. We demonstrate that this approach allows one to determine the phase of the spectral amplitude of this process from the measured cross sections, which is a step toward the so-called complete photoionization experiment [15]. This circumstance is particularly important when electron correlation plays a significant role in the ionization process.

\section{THEORY AND RESULTS}

The part of the wave function that is relevant to the DPI process and, therefore, of interest to us can be expanded over the set of the double continua states of the helium atom:

$\Psi_{1}\left(\boldsymbol{r}_{1}, \boldsymbol{r}_{2}, t\right)=\int d \boldsymbol{q}_{1} d \boldsymbol{q}_{2} f\left(\boldsymbol{q}_{1}, \boldsymbol{q}_{2}\right) \Psi_{\boldsymbol{q}_{1}, \boldsymbol{q}_{2}}^{-}\left(\boldsymbol{r}_{1}, \boldsymbol{r}_{2}\right) e^{-i E t}$,

where $E\left(q_{1}, q_{2}\right)=q_{1}^{2} / 2+q_{2}^{2} / 2$. In the following, we consider the DPI process driven by an XUV pulse with a low intensity. In this case, the amplitudes $f\left(\boldsymbol{q}_{1}, \boldsymbol{q}_{2}\right)$ can be computed perturbatively. The well-known lowest-order perturbation theory (LOPT) result is

$$
f\left(\boldsymbol{q}_{1}, \boldsymbol{q}_{2}\right)=-i \int_{-\infty}^{\infty}\left\langle\Psi_{\boldsymbol{q}_{1}, \boldsymbol{q}_{2}}^{-}\left|\hat{H}_{\mathrm{int}}^{\mathrm{XUV}}\right| \Psi_{0}\right\rangle e^{i\left[E\left(q_{1}, q_{2}\right)-E_{0}\right] \tau} d \tau,
$$

where $\Psi_{0}$ describes the ground state of the helium atom and $\hat{H}_{\text {int }}^{\text {XUV }}$ describes interaction of the atom and the XUV pulse.

A similar perturbative treatment can be applied in the case wherein the helium atom interacts with the XUV and IR fields of low intensity. Technically, however, it is much simpler to treat this case by solving the time-dependent Schrödinger equation (TDSE) for the helium atom in the presence of the XUV and IR pulses. In this paper, particular values of the pulse parameters are chosen as follows.

The time dependence of the electric field of the XUV pulse is $\boldsymbol{E}_{\mathrm{XUV}}(t)=\boldsymbol{E}_{\mathrm{XUV}}^{0} f(t) \cos \omega t$, with the base frequency $\omega=3.307$ a.u. (corresponding to the photon energy of $90 \mathrm{eV}$ ) and the peak field strength $E_{\mathrm{XUV}}^{0}=0.1$ a.u. (corresponding to the intensity of $\left.3.5 \times 10^{14} \mathrm{~W} / \mathrm{cm}^{2}\right)$. The XUV pulse is switched off outside the interval $(-6 T, 6 T)$, where $T=2 \pi / \omega$ is an optical cycle corresponding to the angular frequency of the XUV pulse. The total duration of the XUV pulse is thus $12 T$. The envelope function $f(t)$ for the XUV pulse is chosen in such a way that the amplitude of the XUV field is ramped on and off smoothly during one optical cycle $T$, and is constant in between. Time dependence of the electric field for the IR 
pulse is $\boldsymbol{E}_{\mathrm{IR}}(t)=\boldsymbol{E}_{\mathrm{IR}}^{0} \sin \Omega(t+\Delta)$, with the base frequency $\Omega=0.0367$ a.u. (photon energy of $1 \mathrm{eV}$ ) and peak field strength $E_{\mathrm{IR}}^{0}=0.001$ a.u. The total duration of the IR pulse is $T_{1}$, where $T_{1}=2 \pi / \Omega$ is an optical cycle corresponding to the IR frequency $\Omega$. The IR field is zero outside the interval $\left(-\Delta, T_{1}-\Delta\right)$. Parameter $\Delta$ describes delay between the XUV and IR pulses, which is the time elapsed between the beginning of the IR pulses and the arrival of the center of the XUV pulse. XUV and IR pulses are linearly polarized along the same direction, which we assume to be the $z$ axis.

We solve numerically the TDSE for the helium atom interacting with the XUV and IR fields described above:

$$
i \partial \Psi / \partial t=\left[\hat{H}_{\text {atom }}+\hat{H}_{\text {int }}(t)\right] \Psi,
$$

where $\hat{H}_{\text {atom }}$ is the Hamiltonian of a field-free atom and operator $\hat{H}_{\text {int }}(t)$ describes the electromagnetic (EM) interaction. We choose the velocity form for this operator

$$
\hat{H}_{\text {int }}(t)=\boldsymbol{A}(t) \cdot\left(\hat{\boldsymbol{p}}_{1}+\hat{\boldsymbol{p}}_{2}\right),
$$

with the vector potential

$$
\boldsymbol{A}(t)=-\int_{-\infty}^{t}\left[\boldsymbol{E}_{\mathrm{XUV}}(\tau)+\boldsymbol{E}_{\mathrm{IR}}(\tau)\right] d \tau .
$$

We discretize the Hamiltonian operator on a spatial grid.

For the present problem, we have to solve the TDSE several times for a large interval of time ( 90 cycles of the XUV field). This makes this problem rather computationally demanding. To make it less time consuming, we had to use a rather large grid step size of $\Delta r=0.15$ a.u. The ground state of the helium atom was found using the relaxation procedure. For the grid step size we used, the ground-state energy was -2.806 a.u.

The wave function is represented as a superposition

$$
\Psi\left(\boldsymbol{r}_{1}, \boldsymbol{r}_{2}\right)=\sum_{l_{1}, l_{2}, J} f_{l_{1} l_{2}}^{J}\left(r_{1}, r_{2}\right)\left|l_{1}(1) l_{2}(2) L\right\rangle .
$$

Here, the notation $\left|l_{1}(1) l_{2}(2) \quad L\right\rangle$ is used for bipolar harmonics [16], the functions $f_{l_{1} l_{2}}^{J}\left(r_{1}, r_{2}\right)$ are defined on the radial grid, and summation is restricted to $l_{1}, l_{2}=0-3$ with the total angular momentum $J=0-2$. The range of the angular momenta values included in the Eq. (6) was again dictated by the considerations of the computational cost. Restricting summations in Eq. (6) to $l_{1}, l_{2}=0-3$ allowed us to make the problem computationally tractable, allowing us at the same time to provide a sufficiently accurate description of DPI. Such a range of angular momenta is sufficient to achieve convergence with respect to angular momenta in the calculations of DPI in helium, as was shown in [17], where a computational procedure very similar to ours has been employed. To conclude this discussion of the limitations imposed on us by the computational problems, we should mention that our choice of the IR pulse (pure sine form with one cycle pulse duration) was also motivated by the computational cost considerations. Calculations for the IR pulse with pulse duration of several cycles would have been considerably more time consuming.

Time propagation of the solution of the TDSE was performed using the Arnoldi-Lanczos method [18,19]. At the moment of time when both pulses are gone, the solution of the TDSE is projected on the set of the states $\Psi_{\boldsymbol{q}_{1}}^{-}, \boldsymbol{q}_{2}$ representing the doubly ionized states. As in Eq. (1), these states are described by means of the wave functions which are products of two Coulomb waves with the nuclear charge $Z=2$. Such wave functions (also known as $\mathrm{C} 2$ wave functions in the literature [20]) do not, therefore, include correlation between electrons. At first glance, it seems not quite clear as to why we can hope to describe double ionization of helium accurately using these states. It has been shown, however [21], that projecting a solution of TDSE on the set of the C2 states produces results for total and differential cross sections of DPI in helium and in the even more challenging problem of DPI in hydrogen molecule, in excellent agreement with other theoretical and experimental works. The rationale behind this apparent success of describing final states using uncorrelated wave functions is the observation that, if we allow the system to evolve far enough in time, so that electrons are at large distance from the nucleus and each other, projection on uncorrelated states can produce valid results. More exactly, we can expect this projection technique to be valid if, at the moment of time when we perform the projection operation, kinetic energy of electrons is much larger than their potential energy [21]. This is certainly true in our case. For the calculations reported below, the projection operation is performed at least 30 cycles (corresponding to the angular frequency of the XUV pulse) after the end of the XUV pulse, which drives the ionization process. It is easy to see that, for the excess energy available to electrons in our problem, the interval of time the electrons are allowed to travel, and typical case of approximately equal energy sharing, electron kinetic energy is indeed several times larger than their potential energy.

The projection operation discussed above allows us to compute the DPI amplitude which, after some integrations, can be converted into various probability distributions.

The distribution, which is presently of interest to us, is the momentum distribution of the photoelectrons for the particular escape geometry in which one electron (with the momentum $k_{z}$ ) exits along the $z$ axis, and another (with momentum $k_{x}$ ) along the $x$ axis. In this configuration, it is only the photoelectron escaping in the $z$ direction that feels a noticeable effect of the IR pulse.

The photoelectron momentum distributions described above are presented in Fig. 1. The top left panel corresponds to the DPI process driven by the XUV pulse alone, while other panels visualize the combined effect of XUV and IR pulses with a varying time delay. We clearly see a characteristic ringlike structure along the circle $k_{x}^{2}+k_{z}^{2}=$ const, reflecting the energy conservation. In the presence of the IR pulse, this structure is split and distorted due to possibility of absorption or emission of an IR photon. The effect of the IR field on the spectrum depends quite sensitively on the delay between the XUV and IR pulses. This is hardly surprising as the duration of the IR pulse is only one optical cycle, which necessarily introduces a good deal of asymmetry into the spectra.

By comparing the DPI spectrum driven by the XUV pulse alone with the analogous spectra obtained in presence of both XUV and IR pulses, we can get some information about interference of the processes with participation of the XUV and IR photons. Such an interference is sensitive to the phase of the DPI amplitude. To retrieve the phase, we proceed as follows. 

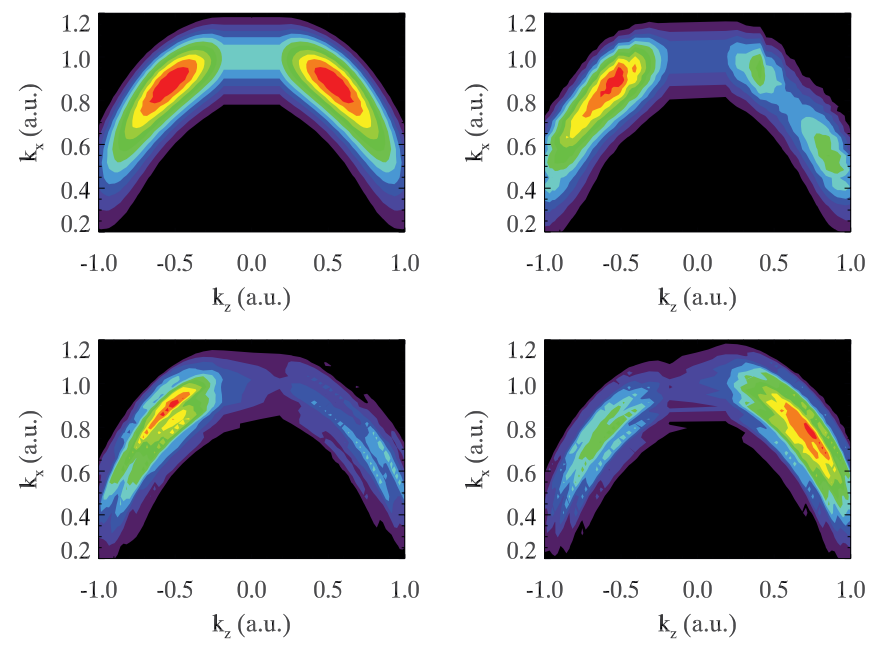

FIG. 1. (Color online) Distribution $\left|f\left(k_{x}, k_{z}\right)\right|^{2}$ for DPI of helium driven by the XUV pulse alone (top left), and in the presence of the IR field for $\Delta=6$ (top right), $\Delta=28 T$ (bottom left), and $\Delta=50 T$ (bottom right). $T$ is the optical cycle for the XUV field.

The problem we are considering belongs to the class of the so-called laser-assisted phenomena. Such phenomena, be it laser-assisted scattering or photoionization, can be described theoretically using the concept of the so-called "dressed" states, which take into account the effect of the IR field. It is these states, not the states $\Psi_{\boldsymbol{q}_{1}, \boldsymbol{q}_{2}}^{-}$entering Eq. (1), that we have to use as the building blocks of our theory.

To put this idea in a quantitative framework, we can use a perturbative expansion for the evolution operator $\hat{U}(t,-\infty)$ describing evolution of the atom in the presence of both XUV and IR fields. Such expansion can be obtained from the Dyson equations for the propagators, and within the first order of the perturbation theory in the atom-XUV field interaction, it reads as

$$
\hat{U}\left(t, t_{0}\right)=\hat{U}_{0}\left(t, t_{0}\right)-i \int_{-\infty}^{t} \hat{U}_{0}(t, \tau) H_{\mathrm{int}}^{\mathrm{XUV}}(\tau) \hat{U}_{0}\left(\tau, t_{0}\right),
$$

where $\hat{U}_{0}\left(t, t_{0}\right)$ is the evolution operator for the atom in the presence of the IR field only. For the spectral amplitudes defined in Eq. (1), we then obtain, after XUV and IR pulses are gone,

$$
\begin{aligned}
f\left(\boldsymbol{q}_{1}, \boldsymbol{q}_{2}\right)= & -i \lim _{t \rightarrow \infty} e^{-i E\left(q_{1}, q_{2}\right) t} \int_{-\infty}^{t}\left\langle\hat{U}_{0}(\tau, t) \Psi_{\boldsymbol{q}_{1}, \boldsymbol{q}_{2}}^{-}\right| \\
& \times \hat{H}_{\mathrm{int}}^{\mathrm{XUV}}(\tau)\left|\Psi_{0}\right\rangle e^{-i E_{0} \tau} d \tau .
\end{aligned}
$$

Here, we assumed that the IR field perturbs the initial ground state only slightly (as it does for the IR field parameters we consider), so we can write $\hat{U}_{0}(\tau,-\infty) \mid \Psi_{0}=e^{-i E_{0} \tau} \Psi_{0}$. The state vector $\hat{U}_{0}(\tau, t) \Psi_{\boldsymbol{q}_{1}, \boldsymbol{q}_{2}}^{-}$describes the effect of the IR field on the states $\Psi_{\boldsymbol{q}_{1}, \boldsymbol{q}_{2}}^{-}$we use for the projection operation. Comparing Eqs. (8) and (2), we see that, to describe the IR field effect, the two-electron state $\Psi_{\boldsymbol{q}_{1}, \boldsymbol{q}_{2}}^{-}$should be replaced with the solution of the TDSE in the presence of the IR pulse, which goes into $\Psi_{\boldsymbol{q}_{1}, \boldsymbol{q}_{2}}^{-}$in the limit of vanishing IR field. This is not a simple problem to solve, and we have to adopt an approximation at this stage to make the problem tractable.
We use the so-called Coulomb-Volkov approximation (CVA) $[22,23]$, assuming that

$$
\begin{aligned}
\hat{U}_{0}(\tau, t) \Psi_{\boldsymbol{q}_{1}, \boldsymbol{q}_{2}}^{-}= & \Psi_{\boldsymbol{q}_{1}, \boldsymbol{q}_{2}}^{-} \exp \left(-i \int_{t}^{\tau}\left\{\left[\boldsymbol{q}_{1}+\boldsymbol{A}(x)\right]^{2} / 2\right.\right. \\
& \left.\left.+\left[\boldsymbol{q}_{2}+\boldsymbol{A}(x)\right]^{2} / 2\right\} d x\right),
\end{aligned}
$$

where $\boldsymbol{A}$ is vector potential for the IR field. The CVA approximation is known to describe satisfactorily singleelectron ionization of atoms [22]. It is by no means guaranteed that CVA is always reliable. In fact, CVA is an interpolation between the solution of TDSE describing the motion of an electron in the presence of the EM field only and the solution of the stationary Schrödinger equation in the Coulomb field without an external EM field. As such, it can not always work reliably. It was found that CVA fails in certain processes of the laser-driven electron scattering [24]. It is therefore our first priority to determine if CVA is applicable to the DPI process that we are interested in.

By neglecting in Eq. (9) terms that are quadratic in $\boldsymbol{A}$ (which is permissible for the field parameters of the IR field we consider), expanding exponential as a Fourier series, and using Eq. (8), one can obtain the following relation between the amplitude $f\left(k_{x}, k_{z}\right)$ of DPI in the presence of the XUV pulse only and the amplitude $f^{1}\left(k_{x}, k_{z}, \Delta\right)$ of DPI in the presence of the XUV and IR pulses:

$$
f^{1}\left(k_{x}, k_{z}, \Delta\right)=\sum_{m=-\infty}^{\infty} f\left(k_{x}, k_{z}^{\prime}\right)(-1)^{m} e^{-i m \Omega \Delta} J_{m}\left(\frac{k_{z} E_{\mathrm{IR}}^{0}}{\Omega^{2}}\right) .
$$

Here, $J_{m}(x)$ is the Bessel function and $k_{z}^{\prime}=\sqrt{k_{z}^{2}-2 m \Omega}$, and the notation $f^{1}\left(k_{x}, k_{z}, \Delta\right)$ is used to emphasize that DPI amplitude in the presence of the XUV and IR pulses depends on the delay $\Delta$. Equation (10) is analogous to the formula given in Ref. [14] for the process of Auger decay following absorption of an XUV photon. Equation (10) was derived for the particular DPI kinematics that we chose above: the electron with momentum $k_{z}$ escapes along the direction of the IR polarization vector, while the electron with momentum $k_{x}$ escapes perpendicular to it. Terms with different $m$ in Eq. (10) describe absorption or emission of $m$ IR photons which change the momentum $k_{z}$ appropriately. For the weak IR field that we consider presently, only the terms with $m=0, \pm 1$ are contributing. This formula, if applicable, would provide us with a simple description of the effect of the IR pulse on the DPI spectrum.

The electron momentum distributions produced by Eq. (10) are shown in Fig. 2. One can see that CVA does reproduce some important features of the TDSE spectrum shown in Fig. 1. In particular, a considerable asymmetry in the $z$ direction due to the IR field is clearly visible. To get a more detailed comparison, we take horizontal slices of the distributions shown in Figs. 1 and 2 for a particular value of $k_{x}=0.9$ a.u. Such a comparison is shown in Fig. 3. One can see that CVA reproduces rather well an overall behavior of the spectra produced by the TDSE calculation.

Armed with this fact, we can extract phases of the amplitudes $f\left(k_{x}, k_{z}\right)$ of the DPI process driven by the XUV pulse alone from the absolute values of the amplitudes 

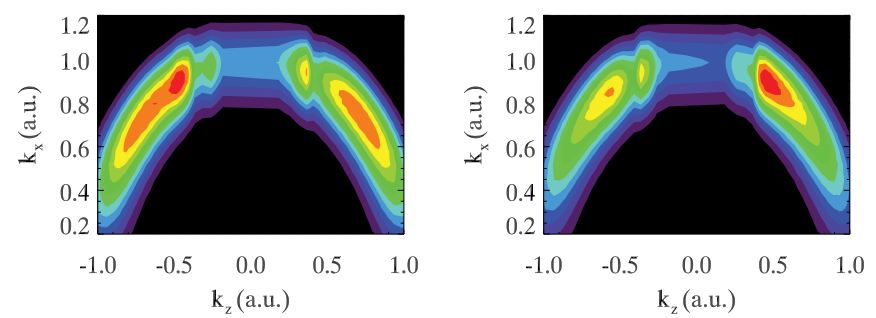

FIG. 2. (Color online) Distribution $\left|f\left(k_{x}, k_{z}\right)\right|^{2}$ for DPI of He in the presence of XUV and IR fields produced by Eq. (10). The time delay between the XUV and IR pulses is is $\Delta=6 T$ (left) and $\Delta=$ $50 T$ (right).

$f^{1}\left(k_{x}, k_{z}\right)$ of DPI in the presence of the XUV and IR fields. This can be done using a procedure similar to the one employed in Ref. [14] for the process of single-electron photoionization followed by Auger decay, or else using the following procedure (which we actually employed).

By using the facts that only the terms with $m=0, \pm 1$ can be retained in Eq. (10) and the difference of arguments $k_{z}$, $k_{z}^{\prime}$ in the amplitudes on the left- and right-hand sides of this equation is small, one can write

$$
\begin{aligned}
& \left|f^{1}\left(k_{x}, k_{z}, \Delta\right)\right|^{2} \\
& \quad=\left|f\left(k_{x}, k_{z}\right)\right|^{2}\left|\sum_{m=-1}^{1} A(\Delta, m)[1+k(\Delta, m) B]\right|^{2},
\end{aligned}
$$
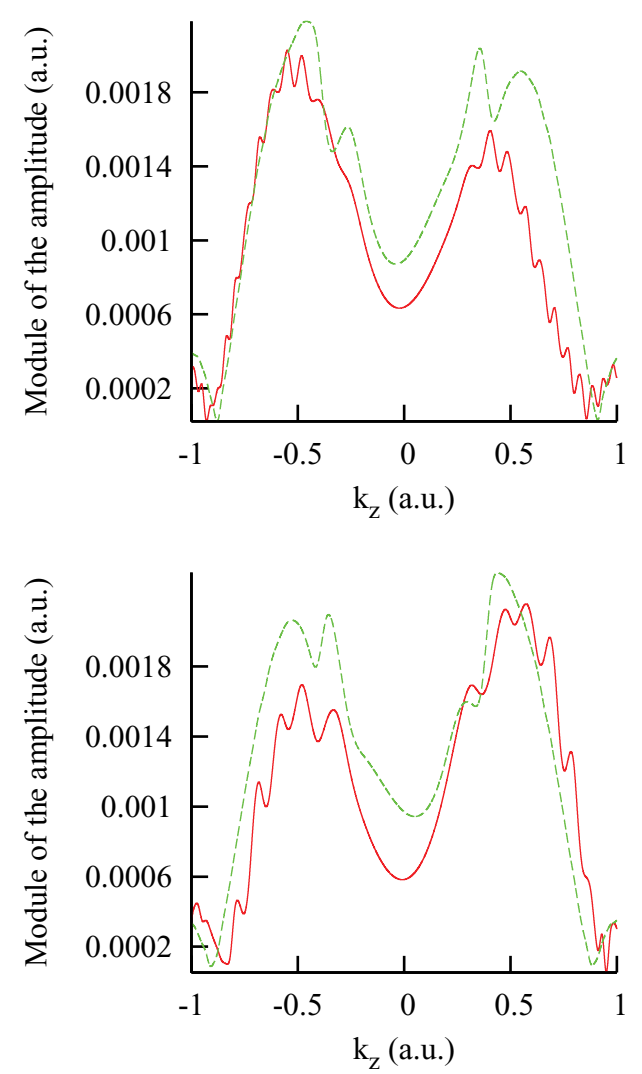

where $A(\Delta, m)=(-1)^{m} e^{-i m \Omega \Delta} J_{m}\left(\frac{k_{z} E_{\mathrm{I}}^{0}}{\Omega^{2}}\right), \quad k(\Delta, m)=k_{z}^{\prime}-$ $k_{z}=\sqrt{k_{z}^{2}-2 m \Omega}-k_{z}$, and $B=f\left(k_{x}, k_{z}\right)^{-1} \frac{\partial f\left(k_{x}, k_{z}\right)}{\partial k_{z}}$. The only unknown quantity in this equation is $B$. By writing down Eq. (11) for several values of $\Delta$, we obtain a system of equations from which $B$ can be determined. In our calculation, we used equations for $\Delta=6 T, 28 T$, and $50 T$, and solved the resulting system of nonlinear equations in the least-squares sense, requiring that the sum of the squared differences of the left- and right-hand sides of Eq. (11) taken over different $\Delta$ values used in the calculation attained its minimum value. We checked the consistency of this recipe, verifying that different choices of the set of parameters $\Delta$ used for constructing a system of equations from which $B$ is found does not affect the results in a significant way.

This procedure allows us to determine parameter $B$ in Eq. (11), which gives us the absolute value of the derivative $\partial f\left(k_{x}, k_{z}\right) / \partial k_{z}$ and its phase relative to the phase of the amplitude $f\left(k_{x}, k_{z}\right)$. Knowing these parameters, we can find the phase of the amplitude $f\left(k_{x}, q\right)$ relative to the phase of $f\left(k_{x}, k_{z}\right)$ for values of $q$ close to the value $k_{z}$ for which the system of equations based on Eq. (11) has been solved. In this way, by moving along the slice $k_{x}=$ const, we can reconstruct the phases of the amplitude $f\left(k_{x}, k_{z}\right)$ for different values of $k_{z}$ relative to some starting value.

The results of this procedure are shown in Fig. 4. Instead of "experimentally observable" $\left|f^{1}\left(k_{x}, k_{z}\right)\right|$, we used the results provided by the TDSE solution which we presume to be nearly
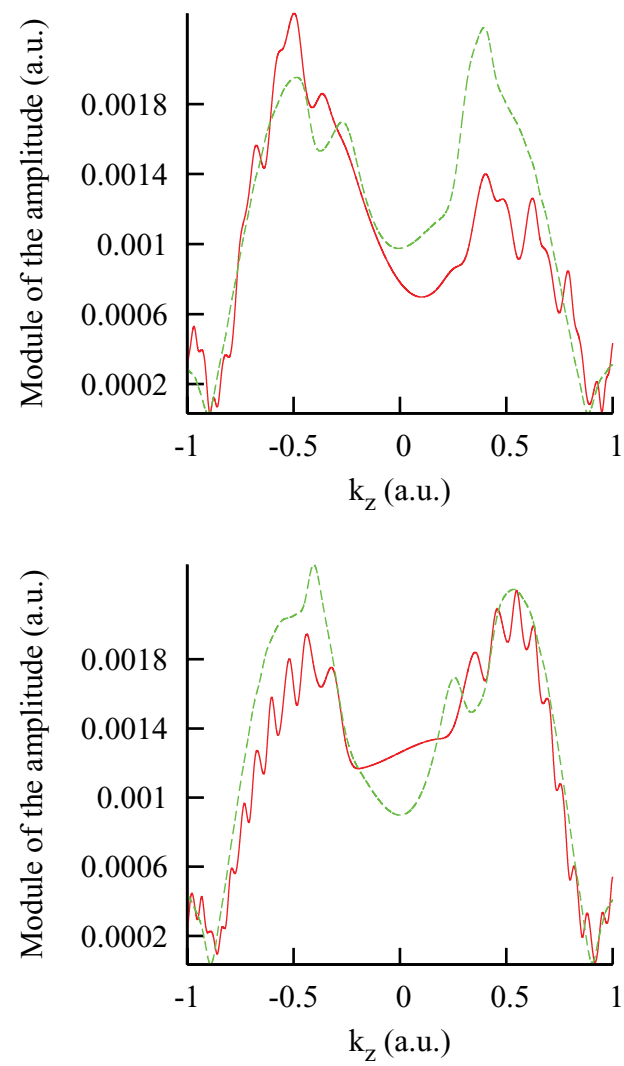

FIG. 3. (Color online) Distribution $\left|f\left(k_{x}, k_{z}\right)\right|^{2}$ as a function of $k_{z}$ for the fixed value $k_{x}=0.9$ a.u. is shown for various delays between the XUV and IR pulses. $\Delta=6 T$ (top left), $\Delta=28 T$ (top right), $\Delta=50 T$ (bottom left), and $\Delta=74 T$ (bottom right). The solid (red) line displays the TDSE calculation, the dashed (green) line corresponds to the CVA result. 

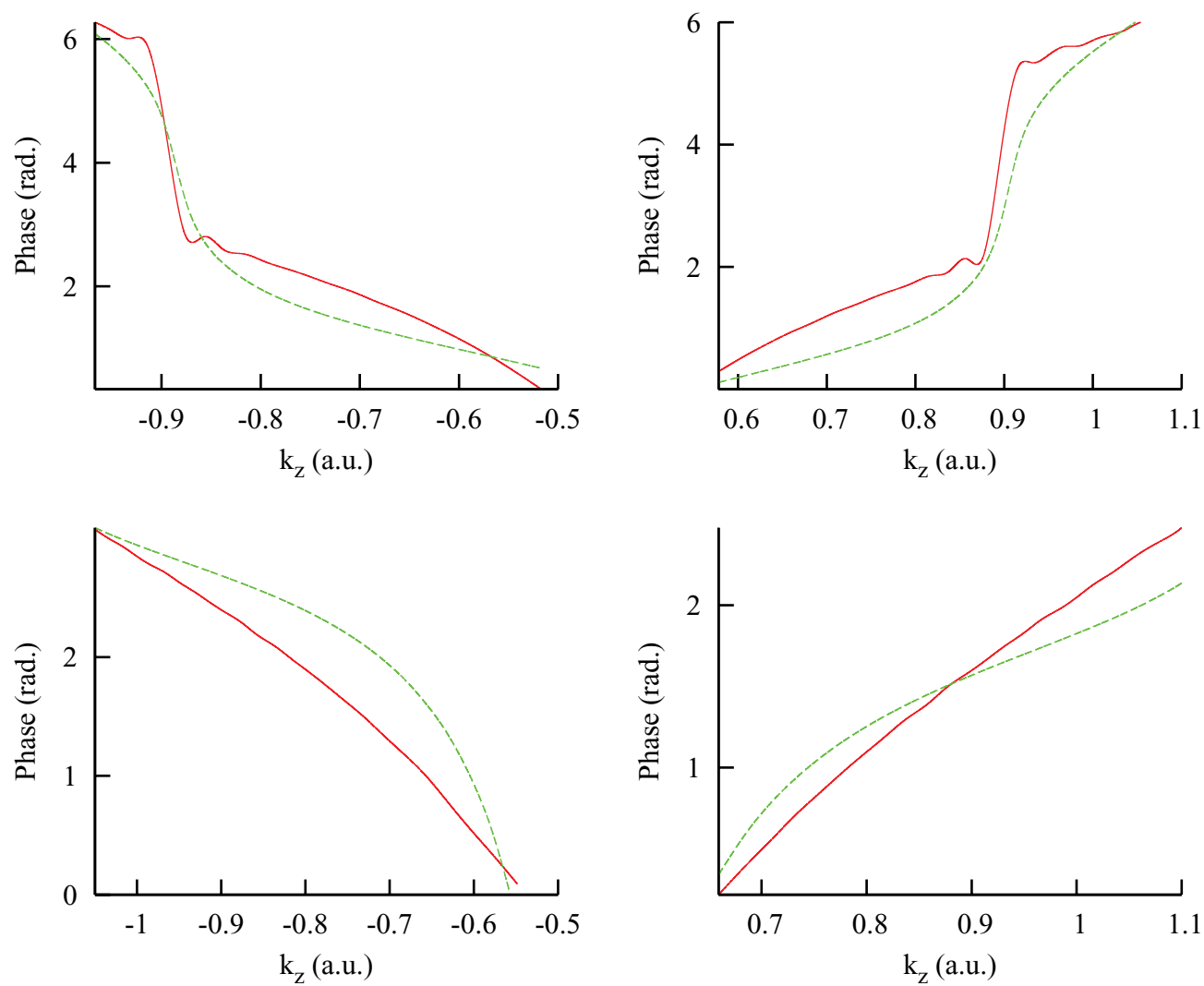

FIG. 4. (Color online) Phase of the amplitude $f\left(k_{x}, k_{z}\right)$ relative to the phase of $f\left(k_{x},-1\right)$ (left panels) and $f\left(k_{x}, 0.6\right)$ (right panels) for $k_{x}=0.9$ a.u. (upper panels) and $k_{x}=0.4$ a.u. (lower panels). Results given by the TDSE calculation for DPI driven by the XUV pulse alone are shown by the (red) solid line. Results obtained by using Eq. (10) are displayed by the (green) dashed line.

as good as experiment. Before discussing these results, we should clarify an important point. Spectral amplitudes of DPI get their full meaning only when a corresponding set of doubly ionized states is defined. By changing arbitrarily phases of the doubly ionized states $\Psi_{\boldsymbol{q}_{1}, \boldsymbol{q}_{2}}^{-}\left(\boldsymbol{r}_{1}, \boldsymbol{r}_{2}\right)$ in Eq. (1), we introduce corresponding changes into the amplitudes $f\left(\boldsymbol{q}_{1}, \boldsymbol{q}_{2}\right)$. Two sets of the states $\Psi_{\boldsymbol{q}_{1}, \boldsymbol{q}_{2}}^{-}\left(\boldsymbol{r}_{1}, \boldsymbol{r}_{2}\right)$, which differ by an arbitrary phase factor, are completely equivalent, and no experiment can possibly tell the difference between them. It is not clear at first sight as to which set of phases the procedure based on Eq. (10) actually provides. The answer to this question can be found after a closer inspection of this equation. Its derivation assumes that a set of states $\Psi_{\boldsymbol{q}_{1}, \boldsymbol{q}_{2}}^{-}\left(\boldsymbol{r}_{1}, \boldsymbol{r}_{2}\right)$ is such that $\Psi_{\boldsymbol{q}_{1}, \boldsymbol{q}_{2}}^{-}\left(\boldsymbol{r}_{1}, \boldsymbol{r}_{2}\right) \approx \Psi_{\boldsymbol{q}_{1}, \boldsymbol{q}_{2}^{\prime}}^{-}\left(\boldsymbol{r}_{1}, \boldsymbol{r}_{2}\right)$, when $\left|\boldsymbol{q}_{2}-\boldsymbol{q}_{2}^{\prime}\right|<\sqrt{2 \Omega}$, which restricts considerably the freedom of choice of the arbitrary phase factor for the set of the doubly ionized states. This factor can not vary too fast, remaining approximately constant on the momentum scale of $\sqrt{\Omega}$.

In Fig. 4, we show results for the phase of the amplitude $f\left(k_{x}, k_{z}\right)$ relative to the two starting values of $k_{z}=-1$ a.u. (left) and $k_{z}=0.6$ a.u. (right) obtained for two horizontal slices of the momentum distributions shown in Figs. 1 and 2. One can see that the phases obtained by the TDSE solution corresponding to DPI driven by the XUV pulse, and the phases obtained using the procedure based on Eq. (10) agree quite well in the intervals of momenta shown in Fig. 4. This procedure works not so reliably for the values of $k_{z}$ outside these intervals. This is not surprising given the fact that the intensity in Fig. 1 is much weaker in this part of the momentum distribution.

\section{CONCLUSION}

We have applied the phase reconstruction technique to the spectral amplitude of DPI of helium. This technique is based on the analysis of the correlated two-electron ionization spectrum. We demonstrated that the phase of the amplitude (relative to an arbitrary starting value) could be determined reliably in a wide interval of photoelectron momenta. This suggests that information about the wave function describing the DPI process, and its temporal development, can be obtained from experimental data. Accurate phase reconstruction of the spectral amplitude and its momentum dependence is also crucial for evaluating the time delay in the photoionization process (this quantity can be related to the energy derivative of phase of the spectral amplitude [25]). This subject received recently a considerable attention after unexpected, and not yet fully understood, results [11] were published, demonstrating anomalously large time delays in photoionization from the neon atom. The problem we consider is not so dissimilar to the problem of the reconstruction of the phase of the scattering amplitude from the experimental data, which is particularly important in nuclear physics. Solution of this problem is 
an important step toward solution of the inverse problem in nuclear physics, i.e., reconstruction of the (unknown) potential from the experimental data [26]. In atomic physics, if we use a completely $a b$ initio approach, the potential is known quite reliably, of course. However, if complex systems are considered, such as the $\mathrm{Ne}$ atom, we necessarily have to use approximations, e.g., the single active electron approximation. In some cases, this may lead to considerable disagreement with experiment. An attempt to resolve this issue, which we undertook in [12] using the random-phase approximation with exchange (RPAE) [27] to partly account for the correlation effects, showed that RPAE can not solve this problem as well. This tells us that electron correlations may play a very important role in ionization phenomena for some atomic systems.
Another example of a strongly correlated phenomenon is the single-photon DPI process, which we considered above. The described technique can help to resolve experimentally electron correlation in time for the DPI in helium. The advantage of the proposed method is that it does not require a sequence of intense XUV pulses needed for the direct XUV pump-probe measurement proposed in Ref. [28].

\section{ACKNOWLEDGMENTS}

The authors acknowledge support of the Australian Research Council in the form of the Discovery Grant No. DP0771312. Resources of the National Computational Infrastructure (NCI) Facility were employed.
[1] A. Baltuška et al., Nature (London) 421, 611 (2003).

[2] P. B. Corkum and F. Krausz, Nat. Phys. 3, 381 (2007).

[3] F. Krausz and M. Y. Ivanov, Rev. Mod. Phys. 81, 163 (2009).

[4] R. Kienberger, E. Goulielmakis, M. Uiberacker, A. Baltuska, V. Yakovlev, and F. Bammer, Nature (London) 427, 817 (2004).

[5] M. Uiberacker et al., Nature (London) 446, 6627 (2007).

[6] S. X. Hu and L. A. Collins, Phys. Rev. Lett. 96, 073004 (2006).

[7] T. Morishita, S. Watanabe, and C. D. Lin, Phys. Rev. Lett. 98, 083003 (2007).

[8] M. E. Kellman and D. R. Herrick, J. Phys. B: At. Mol. Phys. 11, L755 (1978).

[9] C. D. Lin, Adv. At. Mol. Phys. 22, 77 (1986).

[10] J. Mauritsson et al., Phys. Rev. Lett. 105, 053001 (2010).

[11] M. Schultze et al., Science 328, 1658 (2010).

[12] A. S. Kheifets and I. A. Ivanov, Phys. Rev. Lett. 105, 233002 (2010).

[13] K. Klünder et al., e-print arXiv:1012.3863.

[14] O. Smirnova, V. S. Yakovlev, and M. Ivanov, Phys. Rev. Lett. 94, 213001 (2005).

[15] N. A. Cherepkov and S. K. Semenov, J. Phys. B: At., Mol. Opt. Phys. 37, 1267 (2004).
[16] D. A. Varshalovich, A. N. Moskalev, and V. K. Khersonskii, Quantum Theory of Angular Momentum (World Scientific, Singapore, 1988).

[17] J. Colgan, M. S. Pindzola, and F. Robicheaux, J. Phys. B: At., Mol. Opt. Phys. 34, L457 (2001).

[18] T. J. Park and J. C. Light, J. Chem. Phys. 85, 5870 (1986).

[19] D. Dundas, Phys. Rev. A 65, 023408 (2002).

[20] P. Guang-yan, P. Hvelplund, H. Knudsen, Y. Yamazaki, M. Brauner, and J. S. Briggs, Phys. Rev. A 47, 1531 (1993).

[21] J. Colgan, M. S. Pindzola, and F. Robicheaux, J. Phys. B: At., Mol. Opt. Phys. 37, L377 (2004).

[22] G. Duchateau, E. Cormier, and R. Gayet, Phys. Rev. A 66, 023412 (2002).

[23] A. S. Kornev and B. A. Zon, J. Phys. B: At., Mol. Opt. Phys. 35, 2451 (2002).

[24] J. Gagnon, F. Krausz, and V. S. Yakovlev, Phys. Rev. A 82, 033435 (2010)

[25] E. P. Wigner, Phys. Rev. 98, 145 (1955).

[26] V. Kostrykin and R. Schrader, Lett. Math. Phys. 48, 197 (1999).

[27] M. Y. Amusia, Atomic Photoeffect (Plenum, New York, 1990).

[28] S. X. Hu and L. A. Collins, J. Mod. Opt. 54, 943 (2007). 